\title{
Informatização da informação: a experiência do Sistema de Bibliotecas da Universidade Federal do Rio Grande do Sul ${ }^{*}$
}

Eliane Maria Severo Gonçalves Janise Silva Borges da Costa Laís Freitas Caregnato Tânia Marisa de Abreu Fraga

\section{Resumo}

O Sistema de Bibliotecas da Universidade Federal do Rio Grande do Sul vem desenvolvendo, desde 1988, o Sistema de Automação de Bibliotecas da UFRGS $S A B i$. Este trabalho apresenta o SABi, enfatizando os recursos utilizados na sua definição e implementação, sua estrutura e sua aplicação no tratamento, recuperação e difusão dos dados bibliográficos de documentos produzidos no âmbito da universidade.

\section{Palavras-chave}

Informatização; Bibliotecas universitárias; Sistema de bibliotecas; UFRGS; Base de dados; SABi; Produção intelectual; Automação.

\section{INTRODUÇÃO}

A preocupação com o aperfeiçoamento dos serviços bibliotecários oferecidos à comunidade universitária e o surgimento das novas tecnologias da informação determinaram o início do processo de informatização das funções das bibliotecas da Universidade Federal do Rio Grande do Sul (UFRGS), a partir da década de 70. A integração de informações do acervo da universidade, em uma base de dados única, identificada como Sistema de Automação de Bibliotecas (SABi), é um dos principais resultados deste processo.

A importância desta base para o alcance dos objetivos da universidade, consignados no trinômio ensino, pesquisa e extensão, pode ser compreendida explicitando-se as características da UFRGS e de seu Sistema de Bibliotecas.

\section{Universidade Federal do Rio Grande do Sul - UFRGS}

A UFRGS foi fundada em 1934, a partir da fusão de unidades privadas de ensino superior, fundadas no final do século passado. Foi federalizada em 1950 e, em 1965, recebeu a atual denominação de Universidade Federal do Rio Grande do Sul. Está situada no extremo sul do Brasil, no estado do Rio Grande do Sul, na cidade de Porto Alegre. A Universidade conta, atualmente, com 27 unidades distribuídas em quatro campi, localizados em diferentes pontos da cidade. Além disso, conta com órgãos em

* Trabalho apresentado no Congreso Internacional sobre Información Electrónica y Bibliotecas Digitales, Heredia, Costa Rica, 23-26 de setembro de 1997. outros municípios do Estado. Oferece 53 cursos de graduação, 53 cursos de mestrado e 35 cursos de doutorado. Seu quadro é formado por 2275 professores e por 3508 servidores técnico-administrativos. O corpo discente é composto por 21638 alunos.

A UFRGS destaca-se no cenário nacional como a segunda universidade do país em produção intelectual, em relação ao total de professores.

\section{Sistema de Bibliotecas da UFRGS - SBU}

O SBU é composto por 30 bibliotecas, sendo 27 bibliotecas setoriais especializadas em diferentes áreas do conhecimento, duas bibliotecas escolares de $1^{\circ}$ e $2^{\circ}$ graus e uma biblioteca central especializada em referência multidisciplinar, totalizando um acervo de 822733 itens de informação.

Cabe destacar que as bibliotecas encontram-se dispersas geograficamente nos quatro campi em que se distribui a UFRGS, com exceção de uma localizada na cidade do Imbé.

A coordenação do SBU é desempenhada pela Biblioteca Central, criada em 1972, cuja finalidade é complementar o acervo das bibliotecas setoriais e traçar a política biblioteconômica para a universidade.

Diante do quadro de dispersão geográfica da UFRGS e da necessidade de otimizar os serviços oferecidos à comunidade universitária, iniciou-se o processo de informatização do SBU. Este trabalho é um relato sucinto desta experiência. 


\section{SISTEMA DE AUTOMAÇÃO DE BIBLIOTECAS DA UFRGS - SABi}

\section{Breve histórico}

A automação dos serviços bibliotecários na UFRGS foi iniciada em 1975 , com a implementação do formato Calco para registro e recuperação de dados bibliográficos de monografias, visando a organizar o Catálogo de Teses. O sistema operava off-line e integrava o acervo de teses e dissertações das várias bibliotecas da universidade. Estas publicações eram encaminhadas para a Biblioteca Central, onde eram catalogadas e, posteriormente, remetidas à biblioteca da área respectiva para inclusão em seu acervo. O produto principal desse sistema era um catálogo impresso. Em 1983, ocorreu sua desativação por falta de equipamentos na universidade.

Em 1988, teve início a segunda fase do processo de automação do SBU. Com o apoio da Financiadora de Estudos e Projetos (Finep) e do Programa Nacional de Bibliotecas Universitárias (PNBU), foi adquirido o equipamento básico necessário para o desenvolvimento e implantação do software que recebeu o nome de Sistema de Automação de Bibliotecas (SABi). Nesta mesma época, - Ministério da Educação e Cultura, por meio da Secretaria de Ensino Superior, financiou a compra de microcomputadores e impressoras para a entrada de dados nas bibliotecas.

Assim, em 1989, foi implantado o SABi, cujo planejamento, desenvolvimento e coordenação estão a cargo da Biblioteca Central e do Centro de Processamento de Dados da UFRGS. O Sistema é resultante do trabalho de uma equipe composta por bibliotecários, analistas de sistemas e programadores oriundos destes dois órgãos.

A automação do registro de publicações periódicas teve início em 1990/1991 com a conversão de cerca de 12 mil registros da base do Sistema Integrado de Publicações Seriadas (SIPS), desenvolvido pelo Instituto Brasileiro de Informação em Ciência e Tecnologia (IBICT). Após a conversão, cada biblioteca setorial ficou responsável pela revisão catalográfica dos dados de seus títulos de periódicos e a Biblioteca Central pela entrada dos dados. Em 1996, a base SIPS/SABi foi transferida para o computador do SBU, tornando-se disponível para consulta on-line.

Em 1995, o Formato SABi foi ampliado, visando a atender o registro de materiais não convencionais que constituem o acervo do SBU, tais como mapas, CD's, disquetes, discos, partituras e diapositivos, entre outros.

Atualmente, o SABi inclui aproximadamente $25 \%$ do acervo da UFRGS. Na maioria das bibliotecas, a entrada de dados é realizada on-line, uma vez que quase todas já estão interligadas à rede da universidade.

\section{Ficha técnica}

O Sistema prevê a automação de todas as funções de bibliotecas universitárias abrangendo seleção e aquisição de documentos, registro, catalogação, classificação, indexação, serviços aos usuários e serviços administrativos.

Em 1996, foi implantada a versão cliente/servidor, visando à integração futura com o banco de dados Sybase, sob o qual foi desenvolvido o Módulo de Controle de Periódicos.

O programa SABi foi desenvolvido em linguagem $\mathrm{C}$. O Formato $\mathrm{SABi}$ é compatível com o Formato IBICT, considerado o padrão nacional de intercâmbio bibliográfico e catálográfico, facilitando a importação e exportação de dados. Surgiu na década de 70, como uma adaptação do Formato MARC. O sistema gerenciador de base de dados utilizado é o CDS/Isis, dada a sua flexibilidade para tratamento de informações textuais. Permite armazenar e manipular registros de tamanho variável, campos múltiplos com número de ocorrências variável e campos optativos.

O software foi inicialmente implementado em versão monousuário e, em 1991, foi lançada a versão multiusuário, em equipamento Elebra MX-850, com sistema operacional VMS. Em 1993, a base foi transferida para uma máquina U6000-65, da Unisys, com sistema operacional Unix.
O Sistema possui uma série de telas de opções, dispostas hierarquicamente, nas quais são relacionadas suas funções. Ao entrar em uma delas, outra tela de opções é apresentada com funções subordinadas à anterior e assim sucessivamente.

\section{Estrutura}

O SABi é composto por módulos independentes que podem ser alterados separadamente. Cada módulo é representado por um nome que identifica o objetivo da função que desempenha.

Estão implantados os módulos de Registro Bibliográfico, Atualização da Base de Dados, Recuperação da Informação, de Salvamento e de Controle de Periódicos (em caráter experimental).

\section{Módulo de Registro Bibliográfico -} Este módulo permite o cadastramento dos documentos, a partir do qual podem ser gerados relatórios de conferência, fichas catalográficas e etiquetas para lombada, registro patrimonial e ficha de empréstimo. Possibilita, também, a geração de registros em meio magnético para exportação.

\section{Módulo de Atualização da Base de Dados - Este módulo utiliza o CDS/} Isis para atualizar o arquivo invertido do Sistema, dando origem aos pontos de acesso para realização de consultas à base SABi.

Módulo de Recuperação da Informação - Por intermédio deste módulo, é executada a recuperação e identificação de documentos, a partir de vários pontos de acesso, e a impressão dos resultados obtidos, de acordo com chaves de classificação predefinidas.

O SABi oferece duas formas de pesquisa na base de dados:

a) Modo Fácil - Apresenta uma tela com campos de busca preestabelecidos: Autor, Título, Assunto, Datas, Idiomas e Bibliotecas. Os termos de busca podem ser precisos ou truncados e informados em qualquer ordem. 
b) Linguagem de Consulta CDS/Isis Para a realização de buscas que requerem estratégias mais complexas, é necessário o uso da linguagem de consulta do Sistema, que permite a combinação de vários pontos de acesso, mediante o uso dos operadores booleanos. Existem, ainda, recursos como truncar palavras, usar operadores de proximidade ou adjacência e qualificadores de campo, os quais permitem limitar a busca a um ou mais campos específicos. Ao selecionar esta opção, é possível acessar telas de ajuda, onde se encontra disponível um manual simplificado dos recursos para elaboração da estratégia de busca.

As pesquisas podem ser realizadas por consulta ao arquivo invertido ou índice, no caso de campo indexado, ou em texto livre, caso o campo não seja indexado.

A opção Pesquisa através do Dicionário permite exibir os pontos de acesso ou entradas do arquivo invertido, possibilitando identificar entradas já atribuídas e suas variantes, atuando como um catálogo de autoridades simplificado.

A utilização deste recurso é recomendada tanto para fins de recuperação como de registro bibliográfico, podendo ser considerada uma terceira forma de pesquisa no $\mathrm{SABi}$.

Módulo de Salvamento - Neste módulo, são efetuados os back-ups dos arquivos sendo, portanto, responsável pela segurança dos dados da base SABi. Desde a implementação da versão multiusuário no SBU, este procedimento é executado pelo CPD.

Módulo de Controle de Periódicos Este módulo destina-se à descrição da coleção de periódicos. No seu desenvolvimento, foi adotado o software Power-Buildere o gerenciador Sybase.

Está previsto que, a partir deste módulo, serão gerados os dados da coleção do SBU para alimentação da base do Catálogo Coletivo Nacional de Publicações Seriadas (CCN), desenvolvida nacionalmente e coordenada pelo IBICT.

\section{QUADRO 1}

\section{Parágrafos para identificação de documentos produzidos na UFRGS}

\begin{tabular}{|c|c|c|}
\hline Parágrafos & Campos & Subcampos \\
\hline 009 & Produção intelectual da instituição & $\begin{array}{l}\text { Unidade/departamento/órgão } \\
\text { Tipo de documento } \\
\text { Curso de pós-graduação } \\
\text { Curso de especialização } \\
\text { Curso de graduação } \\
\text { Órgão financiador }\end{array}$ \\
\hline 591 & Nota de tese ou dissertação & $\begin{array}{l}\text { Grau acadêmico } \\
\text { Instituição de defesa } \\
\text { Data } \\
\text { Local de defesa }\end{array}$ \\
\hline 592 & Orientador de tese ou dissertação & $\begin{array}{l}\text { Nome } \\
\text { Afiliação }\end{array}$ \\
\hline
\end{tabular}

\section{Controle Bibliográfico}

A enfâse na coleta, organização e divulgação da produção intelectual da UFRGS começou em 1992, em uma atividade conjunta do SBU e da Pró-Reitoria Adjunta de Pesquisa.

A partir de então, o SBU prioriza o registro dos dados bibliográficos da produção científica, técnica, artística e administrativa da universidade, a fim de garantir o controle bibliográfico e a disseminação destas informações pelo SABi.

Desde a sua criação, houve a preocupação de adequá-lo aos instrumentos utilizados pelos órgãos nacionais de fomento à pesquisa e responsáveis pelo desenvolvimento científico e tecnológico do país, com o intuito de evitar duplicação de informações.

Para tanto, foram criados parágrafos específicos para identificar documentos produzidos pelo corpo docente, discente e técnico-administrativo, bem como para atender às necessidades internas da Instituição (quadro 1).

O SABi possibilita a classificação dos registros bibliográficos da produção científica, técnica, artística e administrativa, ordenando-os por:

- tipo de documento, data de publicação e autor;

- autor e data de publicação;

- autor e título.
Os relatórios estatísticos gerados a partir do SABi fornecem o total absoluto e respectivos percentuais. São eles:

- total da produção da UFRGS por unidade/departamento/órgão e tipo de documento;

- total da produção da UFRGS por data de publicação e tipo de documento;

- produção de unidades/departamentos/ órgãos por data de publicação e tipo de documento;

- produção de cursos de pós-graduação (mestrado e doutorado) por tipo de documento;

- produção de cursos de especialização por tipo de documento;

- produção de cursos de graduação por tipo de documento.

Estes recursos possibilitam a confecção de catálogos da produção da universidade, além de fornecer informações para a elaboração de relatórios, projetos, curriculum vitae e dados quantitativos que podem se constituir em indicadores de desempenho da comunidade universitária e subsidiar as atividades gerenciais. 


\section{Difusão da Informação}

A automação, no SBU, possibilitou reunir, em uma única base de dados, os recursos informacionais dispersos fisicamente nas várias bibliotecas que o compõem, facilitando e tornando mais rápido e ágil o processo de identificação e localização de documentos pela comunidade universitária.

Desde 1994, quando a base SABi tornou-se disponível para acesso remoto, por intermédio da Internet, estas possibilidades foram ampliadas à comunidade externa à UFRGS, estimulando o intercâmbio e o compartilhamento de seus recursos bibliográficos.

A automação oportuniza, também, a participação em programas cooperativos desenvolvidos regional e nacionalmente, visto que a exportação dos registros pode ser feita de forma automática, sem acarretar qualquer esforço adicional para a equipe que atua nas bibliotecas, contribuindo para maior difusão do acervo existente no SBU.

Recentemente, o SBU integrou-se a uma base de dados de âmbito nacional, denominada Sistema de Informação sobre Teses (Site), coordenada pelo IBICT, tendo enviado, eletronicamente, seus registros de teses e dissertações.

\section{Automation of information: the experience of the library system of the Federal University of Rio Grande do Sul}

\author{
Abstract \\ The Library System of the Federal University \\ of Rio Grande do Sul has been developing \\ the UFRGS Library Automation System - \\ $S A B i$, since 1988. This paper presents SABi, \\ emphasizing the resources used in its \\ definition and implementation, its structure \\ and its application in the treatment, recovery \\ and diffusion of bibliographic data of \\ documents produced in the University.
}

\section{Keywords}

Automation; University libraries; Library system; UFRGS; Database; SABi; Intellectual property; Automation.

\section{CONSIDERAÇÕES FINAIS}

O SABi está em constante desenvolvimento, seja através da implantação de novos módulos ou da implementação de melhorias visando a seu aperfeiçoamento. Neste sentido, encontra-se operando, em caráter experimental, o Módulo de Controle de Periódicos, nas bibliotecas do Centro de Processamento de Dados, do Instituto de Química, da Escola de Engenharia e da Faculdade de Agronomia.

Fatores como integração, padronização, compatibilidade e intercâmbio de informações norteiam a atividade do SBU no que diz respeito à sua informatização, influenciando, ao longo destes anos, o aprimoramento profissional da equipe que atua nas bibliotecas.

Cabe destacar, ainda, que o SABi assegura a preservação da memória institucional e proporciona a ampla difusão da produção intelectual da universidade.

\section{BIBLIOGRAFIA}

1. COSTA, Janise Silva Borges da. Coleta, organização e divulgação da produção in telectual gerada na UFRGS. In: SEMINÁRIO NACIONAL DE BIBLIOTECAS UNIVERSITÁRIAS, 9., 1996. Anais... Curitiba: UFPR, 1996. p. 7.2 .

2. COSTA, Janise Silva Borges da. SABi : Sistema de Automação de Bibliotecas da Universidade Federal do Rio Grande do Sul. In: ENCONTRO NACIONAL DE BIBLIOTECÁRIOS DE ESCOLAS TÉCNICAS AGROTÉCNICAS E CEFETS, 3., 1995. Anais.. Porto Alegre: UFRGS, 1995. p.10-15.

3. SILVA, Elenice Ávila da et al. Automação do Sistema de Bibliotecas da Universidade Federal do Rio Grande do Sul. In: SEMINÁRIO SOBRE AUTOMAÇÃO EM BIBLIOTECAS E CENTROS DE DOCUMENTAÇÃO, 5., 1994. Anais... São José dos Campos: UNIVAP, 1994. p.110-113.

4. UNIVERSIDADE FEDERAL DO RIO GRANDE DO SUL. Pró-Reitoria de Planejamento. Anuário estatístico da UFRGS: 1995. Porto Alegre, $1996.280 p$.

5. Sistema de Bibliotecas. Avaliações de desempenho 1988. Porto Alegre: BC/ UFRGS, 1989. 3v.

6.

Relatório 1992. Porto Alegre: BC/UFRGS, 1993. 3v.

7 Relatório 1993. Porto Alegre: BC/UFRGS, 1994. 2v.

Artigo aceito para publicação em 28-4-98

Eliane Maria Severo Gonçalves*

Bibliotecária - CRB-10/796

Biblioteca Central

E-mail: eliane@vortex.ufrgs.br eliane@bc.ufrgs.br

Janise Silva Borges da Costa*

Bibliotecária - CRB-10/698

Biblioteca da Escola de Engenharia

E-mail: janise@vortex.ufrgs.br

Laís Freitas Caregnato*

Bibliotecária - CRB-10/555

Biblioteca da Faculdade de Agronomia

E-mail: lais@vortex.ufrgs.br

Tânia Marisa de Abreu Fraga*

Bibliotecária - CRB-10/765

Biblioteca da Faculdade de Ciências

Econômicas

E-mail: tania@vortex.ufrgs.br

* Membros do Grupo Assessor Técnico em Informações Eletrônicas do Sistema de Bibliotecas da UFRGS. 